\title{
Desafios no cuidado às complicações agudas do diabetes mellitus em serviço de emergência adulto
}

\author{
Dainai Moraes Oliveira ${ }^{1}$, Soraia Dornelles Schoeller ${ }^{2}$, Karina Almeida Hammerschmidt ${ }^{3}$, \\ Denise Maria Guerreiro Vieira da Silva ${ }^{4}$, Cecília Arruda ${ }^{5}$
}

\footnotetext{
${ }^{1}$ Enfermeira. Discente do Programa de Mestrado Profissional Associado à Residência Médica em Cuidados Intensivos e Paliativos da Universidade Federal de Santa Catarina. Florianópolis, SC, Brasil. Email: oliveiradai2@hotmail.com.

${ }^{2}$ Enfermeira, Doutora em Filosofia da Saúde e Enfermagem. Professora Adjunto da Universidade Federal de Santa Catarina. Florianópolis, SC, Brasil. E-mail: soraia.dornelles@ufsc.br.

${ }^{3}$ Enfermeira, Doutora em Enfermagem. Professora Adjunto da Universidade Federal de Santa Catarina. Florianópolis, SC, Brasil. E-mail: karina.h@ufsc.br.

${ }^{4}$ Enfermeira, Doutora em Enfermagem. Professora Titular da Universidade Federal de Santa Catarina. Florianópolis, SC, Brasil. E-mail: denise.guerreiro@ufsc.br.

${ }^{5}$ Enfermeira, Doutora em Enfermagem. Enfermeira do Hospital Universitário da Universidade Federal de Santa Catarina. Florianópolis, SC, Brasil. E-mail: cecilia2030@gmail.com.
}

Recebido: 21/07/2015. Aceito: 01/12/2015. Publicado: 30/06/2016.

Como citar esse artigo: Oliveira DM, Schoeller SD, Hammerschmidt KA, Silva DMGV, Arruda C. Desafios no cuidado às complicações agudas do diabetes mellitus em serviço de emergência adulto. Rev. Eletr. Enf. [Internet]. 2016 [acesso em: ____ ] 18:e1163. Disponível em: http://dx.doi.org/10.5216/ree.v18.35523.

\section{RESUMO}

O diabetes mellitus impõe múltiplos desafios às pessoas afetadas, suas famílias e ao sistema de saúde. Teve como objetivo analisar fortalezas e fragilidades no atendimento de enfermagem às complicações agudas do diabetes mellitus num serviço de emergência adulto. Pesquisa qualitativa do tipo descritivo-exploratória, realizada entre maio e julho de 2013, mediante entrevistas semiestruturadas com 18 profissionais de enfermagem de um hospital do sul do Brasil. Para organização e análise dos dados, elencaram-se categorias temáticas: estruturação dos serviços de saúde e equipes de trabalho, cada uma com suas fortalezas e fragilidades. Destaca-se que o desafio de qualificar os atendimentos às pessoas com diabetes vai além da estruturação da rede de atenção à saúde e estabelecimento de rotinas assistenciais adequadas a cada instituição, estão aliadas ao conhecimento técnico da equipe profissional, desenvolvimento de habilidades de comunicação, sensibilização sobre a importância do autocuidado e adesão ao tratamento.

Descritores: Enfermagem em Emergência; Diabetes Mellitus; Complicações do Diabetes; Serviço Hospitalar de Enfermagem.

\section{INTRODUÇÃO}

Diabetes mellitus (DM) é condição crônica complexa, caracterizada por hiperglicemia decorrente de alterações na secreção e/ou ação da insulina, que necessita de cuidados em saúde contínuos com uso de estratégias de redução de risco multifatoriais. O diagnóstico é geralmente efetuado com base nos valores de 
glicose no plasma e mais recentemente da hemoglobina glicada (A1C) $)^{(1)}$

O número de pessoas com DM é crescente devido ao aumento populacional com maior expectativa de vida, urbanização, sedentarismo, dieta inadequada e obesidade, assim como maior sobrevida de pessoas com diabetes. O DM, bem como outras doenças crônicas não transmissíveis (DCNT) têm sido responsáveis por elevado número de mortes prematuras, perda de qualidade de vida com alto grau de limitação nas atividades de trabalho e lazer ${ }^{(2-3)}$

A evolução não controlada do diabetes pode levar a complicações agudas e crônicas. As agudas geralmente decorrem de eventos esporádicos, e as crônicas têm origem no mau controle glicêmico ao longo dos anos, sendo ambas responsáveis por elevada morbimortalidade ${ }^{(4)}$.

As principais situações agudas relacionadas ao DM são a hipoglicemia grave com alteração cognitiva e a cetoacidose metabólica $(C A D)^{(4)}$. Há a necessidade de imediata identificação e intervenção pela enfermagem, pois as consequências são graves, variando do coma à morte ${ }^{(5)}$.

Considerando o diabetes com sua elevada carga de morbimortalidade associada é essencial realizar a prevenção no início do DM (prevenção primária); identificação de casos não diagnosticados para tratamento (prevenção secundária); e intensificação do controle de pacientes já diagnosticados visando prevenir complicações agudas e crônicas (prevenção terciária). Para haver eficácia nas ações, os três graus de prevenção devem ocorrer de forma articulada em todos os níveis de atenção à saúde ${ }^{(6-7)}$.

A área de urgência e emergência é importante componente da assistência à saúde. Com objetivo de organizar o sistema de atenção em redes integradas que permitam responder, com efetividade, eficiência, segurança, qualidade e equidade, às condições de saúde da população brasileira, o Ministério da Saúde (MS) estabeleceu as Redes de Atenção à Saúde (RAS). Nesta lógica, os hospitais devem cumprir, principalmente, a função de responder às condições agudas ou aos momentos de agudização das condições crônicas, conforme estabelecido em diretrizes clínicas baseadas em evidências. Para isso, necessitam de densidade tecnológica compatível com o exercício dessa função e padrões de qualidade ${ }^{(8)}$.

As complicações agudas do DM são atendidas nas urgências/emergências hospitalares, cabendo à enfermagem o primeiro contato com o paciente. Neste âmbito a presteza e rapidez do atendimento são essenciais ao prognóstico ${ }^{(9)}$. Nessa perspectiva, com intenção de qualificar o atendimento de enfermagem às complicações agudas do DM, teve-se como objetivo: identificar fortalezas e fragilidades no atendimento de enfermagem às complicações agudas do diabetes mellitus num serviço de emergência adulto.

\section{MATERIAIS E MÉTODOS}

Pesquisa qualitativa do tipo descritivo-exploratória realizada com profissionais de enfermagem (técnicos e enfermeiros) atuantes no serviço de emergência adulto de um hospital do sul do Brasil, no Estado de Santa Catarina. A pesquisa foi realizada atendendo as Normas Brasileiras para Pesquisas com Seres Humanos, com projeto aprovado pelo Comitê de Ética em Pesquisa com Seres Humanos, de CAAE: 13353113.6.0000.0121, com o parecer 252.702, de abril de 2013. 
Participaram do estudo, profissionais integrantes da equipe de enfermagem, lotados no serviço de urgência/emergência do referido hospital. Os critérios de inclusão foram atuação no serviço há pelo menos seis meses. Como critério de exclusão foi considerado estar ausente do serviço durante a coleta de dados. Os participantes foram contatados pessoalmente pela pesquisadora na seguinte ordem: turno matutino, seguido por um do turno vespertino e em sequência por um do noturno. Após aceitarem participar e assinar o TCLE, foram entrevistados pela mesma, em local por eles definidos, sendo todos no próprio serviço, em sala privada. Participaram do estudo 18 profissionais, sendo cinco enfermeiros e 13 técnicos de enfermagem. Para determinar a quantidade de participantes, foi seguido o princípio da saturação dos dados ${ }^{(10)}$, contemplando funcionários de todos os turnos de trabalho: matutino, vespertino e noturno.

A coleta dos dados ocorreu no período de maio a julho de 2013, utilizando entrevista semiestruturada, com roteiro contendo: questões para a caracterização dos profissionais (grau de instrução, categoria profissional, tempo de formação, tempo de atuação no serviço de emergência adulto, experiências profissionais prévias); seguido de perguntas relacionadas à identificação de fragilidades, fortalezas e desafios no atendimento de enfermagem em emergência aos pacientes com complicações agudas do DM - CAD e hipoglicemia severa; e conhecimento sobre rotinas assistenciais da instituição nestes casos e rede de apoio no SUS para pessoas com DM.

As entrevistas gravadas foram realizadas individualmente, em horário escolhido pelo entrevistado. Para a identificação dos participantes, estabeleceu-se o uso da inicial "E" para enfermeiro e "TE" para técnico de enfermagem, atribuídos números sequenciais de acordo com a realização das entrevistas.

Para organização e análise dos dados, seguiram-se os pressupostos metodológicos da Análise Temática segundo proposta de Minayo ${ }^{(11)}$, com a transcrição integral das entrevistas e leitura exaustiva do material empírico. Em seguida, foi efetuada a codificação dos dados para a seleção daqueles que continham as ideias relevantes que constituíram as unidades de significado, as quais foram organizadas em subtemas, relacionando-os às categorias temáticas.

\section{RESULTADOS}

Da análise dos depoimentos emergiram as seguintes categorias temáticas: estruturação dos serviços de saúde e equipes de trabalho, cada uma com suas fortalezas e fragilidades.

$\mathrm{Na}$ categoria estruturação dos serviços de saúde, evidenciaram-se como fortalezas: existência de rotina institucional para hipoglicemia e conhecimento sobre a rede de apoio no SUS às pessoas com DM.

É uma rotina, todo mundo sabe o que deve ser feito no caso da hipoglicemia (E1) Acredito que todos têm conhecimento (TE4).

[...] Tem unidades de pronto atendimento, emergências, ambulatório de diabetes aqui no HU, postos de saúde (E2).

Em relação às fragilidades, foi referenciado: estrutura física inadequada do setor de emergência; falhas 
na rede de atenção à saúde e sobrecarga de trabalho.

A sala de reanimação fica isolada do posto de enfermagem e o posto é pequeno [...](E2).

[...] acompanhamento não tem, os pacientes chegam na emergência com hipoglicemia e CAD. Daqui cinco anos ele aparece com amputação de membro inferior. Então esse paciente não é acolhido, ele está largado na rede, na sorte (E4)

Na emergência, o diabético é bem assistido, o problema é em casa. O acompanhamento dele no posto de saúde (TE3).

[...] na emergência tem poucos funcionários, pelo movimento, acontece tanta coisa ao mesmo tempo e o número elevado de pacientes [...] (TE5).

$\mathrm{Na}$ categoria equipes de trabalho, destacaram-se as fortalezas: conhecimento da rotina institucional para hipoglicemia e realização dos encaminhamentos/contra-referência para a rede de atendimento no SUS aos pacientes com DM.

A rotina é conhecida porque está acessivel, é de praxe [...] (TE12).

Quando vão dar alta, geralmente encaminha-se o paciente para a atenção básica em saúde, com carta e receita (E1)

[...] às vezes encaminha para o ambulatório [...] (E2)

[...] quando é diagnóstico novo ou sem tratamento adequado, agenda-se consulta com as enfermeiras do ambulatório (E3)

Já vi encaminhamentos quando o paciente descobre ser diabético [...] (TE3).

Como fragilidades emergiram: monitorização da glicemia, conhecimento da equipe sobre DM e identificação da situação de emergência.

Às vezes está muito tumultuado e pode não ser feito o hemoglicoteste (HGT), perdendo o controle da glicemia $[\ldots](\mathrm{E} 1)$

o controle frequente da glicose, muitas vezes, passa despercebido (TE4)

[...] na emergência, o controle rigoroso do HGT fica prejudicado pelo movimento do setor com número elevado de pacientes, pouco funcionário, acaba sendo esquecido (TE5).

[...] as pessoas atendem, acabam fazendo porque foi sempre assim, não pensam porque as coisas são feitas

$[\ldots](\mathrm{E} 4)$

A gente percebe diferenças de condução do caso pela medicina, com maior dificuldade dos residentes (E5)

Na CAD, sabemos o básico, mas poderia ser algo mais aprofundado (TE1).

[...] na tutoria daquele curso de especialização [...] eu li o que tem no módulo (E2)

Sim, na faculdade agora (TE4).

Não conheço, nada oficial” (TE5), "Não conheço, nunca ouvi falar (TE3).

[...] paciente já está aqui internado e acaba fazendo hipoglicemia aos nossos olhos e não se percebe (E4) 
Eu acho que a gente tem mais dificuldade para detectar antes, o paciente fica inconsciente e primeiro vamos ver pressão arterial e outras causas e depois pensa na glicemia (TE1).

\section{DISCUSSÃO}

$\mathrm{Na}$ instituição de estudo foi evidenciada a existência de rotina para atendimentos de hipoglicemias, o que é convergente com as indicações para que instituições de saúde tenham protocolos e rotinas estabelecidos $^{(12)}$.

A existência de rede de apoio na atenção primária em saúde (APS) do SUS para acompanhamento das pessoas com DM é fortaleza destacada no atendimento às complicações agudas neste estudo. No Brasil, para corrigir os déficits na atenção à saúde, o governo estabeleceu prioridades para as DCNT, através do "Plano de Ações Estratégicas para o Enfrentamento das DCNT no Brasil, 2011-2022", e tem objetivo de promover o desenvolvimento, implementar políticas públicas efetivas, integradas, sustentáveis, baseadas em evidências para a prevenção, visando controle de seus fatores de risco e fortalecimento dos serviços de saúde voltados para a atenção às pessoas com doenças crônicas ${ }^{(2)}$.

A APS atende aproximadamente $60 \%$ da população brasileira. As equipes atuam em território definido, com população adstrita, realizando ações de promoção, vigilância em saúde, prevenção, assistência, além de acompanhamento longitudinal dos usuários, o que é fundamental para eficácia no tratamento das pessoas com doenças crônicas. Além do acompanhamento regular e sistemático pela equipe de atenção básica, toda pessoa com DM residente no Brasil, cadastrado no SUS tem direito de receber, gratuitamente, todos os insumos e medicamentos para o tratamento da doença ${ }^{(2-3)}$.

Apesar dos avanços e conquistas do SUS, ainda existem grandes lacunas no acesso e acolhimento nos serviços de saúde. Emergiram nas entrevistas algumas fragilidades, referentes ao ambiente, no atendimento às complicações agudas do DM: estrutura física inadequada do serviço de emergência com sobrecarga de trabalho e pouca efetividade das ações de atenção básica.

Encontra-se na literatura relatos das diversas dificuldades a serem superadas no atendimento em saúde nos serviços de emergência: superlotação com dimensionamento insuficiente de profissionais, infraestrutura inadequada, processo de trabalho fragmentado, conflitos e assimetrias de poder, exclusão dos usuários na porta de entrada, desrespeito aos direitos desses usuários, pouca articulação com o restante da rede de serviços, entre outros. Essas situações podem ser identificadas na maioria das unidades públicas de urgência do Brasil e têm interferido consideravelmente no processo de trabalho e na qualidade do cuidado prestado à população(6,13-14).

Para fortalecer a atenção integral e de qualidade no atendimento do DM, é indispensável aumentar a resolutividade na rede básica, reduzindo a necessidade de encaminhamentos para níveis de maior complexidade. É necessária a construção de modelo de atenção aos pacientes com DM, integral e integrada, envolvendo os três níveis de atenção, hierarquizado, centrado no usuário, construído a partir da atenção básica, ficando a média e a alta complexidade responsáveis pelas ações suplementares ou 
complementares ${ }^{(15)}$.

Para o sucesso na implementação de protocolos especiais de controle da hiperglicemia intrahospitalar, além de fundamentação técnica bem definida, é fundamental o desenvolvimento de estratégia informativa e motivacional, abrangendo os diversos segmentos clínicos, administrativos e de especialidades de apoio que compõem a equipe multidisciplinar ${ }^{(16)}$.

Foram relatadas como fragilidades no atendimento às complicações agudas do DM no serviço de emergência, com relação ao paciente com DM, dificuldade de acesso venoso e pouca adesão ao tratamento. Considerando que a inserção de cateteres intravasculares periféricos constitui intervenções frequentemente executadas em pacientes hospitalizados, e que a equipe de enfermagem é responsável pela inserção e manutenção destes nos atendimentos de emergência a pacientes com DM com rede venosa de difícil acesso ou histórico de várias internações prévias, estas podem demonstrar fragilidade para o atendimento imediato que a situação demanda(17).

O controle glicêmico e metabólico no DM constitui desafio para a pessoa com DM, sua família e profissionais de saúde a fim de minimizar complicações em curto e longo prazo. Mudanças comportamentais como seguimento do plano alimentar, monitorização da glicemia, realização de atividade física e adesão ao tratamento medicamentoso são essenciais para prevenção das complicações agudas e crônicas. A não adesão ao tratamento do DM é problema conhecido no cenário nacional e internacional, pois prejudica a resposta fisiológica à doença, a relação profissional-paciente, e aumenta o custo direto e indireto do tratamento ${ }^{(18)}$.

No atendimento de emergência às complicações agudas do DM, destacaram-se como fortalezas, o conhecimento pela equipe de enfermagem da rotina institucional para atendimento de casos de hipoglicemia e a realização de encaminhamentos ou contra-referência para rede básica de saúde no SUS. Porém, os profissionais que atuam no período noturno negaram a realização de contra-referência para a atenção básica em saúde.

A continuidade no acompanhamento da pessoa diabética por toda a rede de saúde é importante para evitar ocorrência e recorrência das complicações agudas e crônicas da doença. Desta maneira, a educação ao paciente que recebe alta hospitalar deve ser direcionada para o reconhecimento dos sinais e sintomas e possíveis causas das descompensações agudas do DM, bem como autocuidado. Desta forma, a equipe de saúde pode desenvolver plano de intervenções que assegure o desenvolvimento de habilidades para o autocontrole da doença ${ }^{(16,19)}$

A literatura ressalta importância de desenvolver articulações com a atenção básica, ambulatórios de especialidade, serviços de atenção e internação domiciliar, entre outros disponíveis na região, visando promover a reinserção do usuário em áreas que qualificam a alta da unidade de emergência, produzindo possibilidades de vínculo e responsabilização(20).

Neste contexto, o profissional enfermeiro tem possibilidade de articular o atendimento e a equipe de saúde. Concorda-se com autores que referem que os profissionais de saúde podem desenvolver práticas 
inovadoras de acordo com o contexto de sua população, buscando a integralidade no seu atendimento ${ }^{(21)}$.

Como fragilidades no atendimento de emergência aos casos agudos de DM, com relação aos profissionais, foram citadas a monitorização da glicemia capilar regular, que, muitas vezes, deixa de ser feita no intervalo preconizado devido demanda do setor; o déficit de conhecimento da equipe sobre DM e também o desconhecimento da maioria dos profissionais sobre as recomendações de órgãos referência no atendimento de casos de DM. Estudos indicam a importância do cuidado precoce por parte da enfermagem em situações de complicações agudas ${ }^{(5)}$, demonstrando que a sobrevivência do paciente depende do reconhecimento precoce dos sintomas de CAD, pronto diagnóstico e manejo adequado ${ }^{(22)}$. Isto requer, além do conhecimento científico necessário, o estabelecimento de um plano de cuidados rigoroso e seguro.

Infelizmente, muitas vezes, os cuidados com o diabetes mellitus são negligenciados, especialmente por aqueles que tendem a não reconhecer a gravidade da mesma. Este fato é decorrente da falta de motivação, descrença na gravidade da doença ou nos benefícios que determinadas ações podem trazer para o seu controle ${ }^{(23)}$. Destaca-se que pessoas com diabetes têm risco aumentado de hospitalização e inclusive de hospitalizações repetidas, em comparação com aquelas sem diabetes, o que afeta negativamente a qualidade de vida do indivíduo além de aumentar o encargo para os serviços de saúde ${ }^{(24)}$.

Por fim é relevante destacar que o aparecimento de complicações observadas em pessoas com mais tempo da doença em interação com maior número de anos vividos pode ser afetado não só pela exposição clínica, mas também pelo tratamento que receberam ao longo da vida ${ }^{(25)}$.

\section{CONSIDERAÇÕES FINAIS}

O estudo apontou que há importantes fragilidades na atenção às pessoas com DM atendidas em serviço de emergência, que incitam o desafio de promover melhorias no atendimento de enfermagem como: implementação de rotinas e protocolos para atendimento às complicações agudas do DM; maior efetividade nas ações de atenção básica para que os casos de hipoglicemia e CAD sejam evitados; promover adesão das pessoas com DM ao tratamento; educação permanente e continuada de toda equipe de saúde.

Há necessidade de qualificação da rede de atenção à saúde, apesar de destacados avanços e conquistas nas políticas públicas de atenção a doenças crônicas, ainda há pontos a serem superados para qualificar os atendimentos de emergência às pessoas com complicações agudas do DM. As mudanças necessárias vão além da estruturação da rede de atenção à saúde e estabelecimento de rotinas assistenciais adequadas a cada instituição, devem estar aliadas ao conhecimento técnico dos profissionais e desenvolvimento de habilidades de comunicação. Também é essencial sensibilizar as pessoas com DM sobre a importância do autocuidado, na tentativa de sucesso na adesão ao tratamento e prevenção das complicações agudas e crônicas.

Cabe salientar que esta pesquisa apresenta como limitação a não possibilidade de generalização, pois se constitui de dados de equipe de enfermagem inserida em uma realidade hospitalar específica. Destaca-se a capacitação clínica dos profissionais como potencial fator ainda em lacunas para futuros estudos, além da 
identificação do perfil dos indivíduos com complicações do diabetes.

\section{REFERÊNCIAS}

1. American Diabetes Association. Standards of medical care in diabetes - 2014. Diabetes Care [Internet]. 2014 [acesso em: 30 jun. 2016];37 Suppl 1:S14-80. Disponível em: http://dx.doi.org/10.2337/dc14-S014.

2. Ministério da Saúde. Plano de ações estratégicas para o enfrentamento das doenças crônicas não transmissíveis (DCNT) no Brasil 2011-2022 [Internet]. Brasília: Ministério da Saúde; 2011 [acesso em: 30 jun. 2016]. Disponível em: http://bvsms.saude.gov.br/bvs/publicacoes/plano acoes_enfrent_dcnt_2011.pdf.

3. Sociedade Brasileira de Diabetes. Diretrizes da Sociedade Brasileira de Diabetes: 2013-2014 [Internet]. São Paulo: AC Farmacêutica, 2014 [acesso em: 30 jun. 2016]. Disponível em: https://www.diabetes.org.br/images/pdf/diretrizessbd.pdf.

4. Dom M, Calder A, Mat R. Características clínico epidemiológicas de las complicaciones agudas de la diabetes en el servicio de urgencias del Hospital General de Atizapán. Rev la Fac Med [Internet]. 2013 [acesso em: 30 jun.

2016];56(2):25-36. Disponível em: http://ref.scielo.org/7tsxnn.

5. Garcia-Pascual MC, Kidby J. Procedures and medications to help patients control their diabetes. Emerg Nurse [Internet]. 2012 [acesso em: 30 jun. 2016];20(8):30-5. Disponível em:

http://dx.doi.org/10.7748/en2012.12.20.8.30.c9481.

6. Ministério da Saúde. Estratégias para o cuidado da pessoa com doença crônica: diabetes mellitus (Cadernos de Atenção Básica, n. 36). Brasília: Ministério da Saúde, 2013 [acesso em: 30 jun. 2016]. Disponível em: http://bvsms.saude.gov.br/bvs/publicacoes/estrategias_cuidado_pessoa_diabetes_mellitus_cab36.pdf.

7. Benamer S, Eljazwi I, Mohamed R, Masoud H, Tuwati M, Elbarsha AM. Association of Hyperglycemia with InHospital Mortality and Morbidity in Libyan Patients with Diabetes and Acute Coronary Syndromes. Oman Med J [Internet]. 2015 [acesso em: 30 jun. 2016];30(5):326-30. Disponível em: http://dx.doi.org/10.5001/omj.2015.67. 8. Mendes EV. As redes de atenção à saúde [Internet]. Brasília: Organização Pan-Americana da Saúde, 2011 [acesso em: 30 jun. 2016]. Disponível em: http://www.conass.org.br/bibliotecav3/pdfs/redesAtencao.pdf.

9. Oliveira DM, Schoeller SD, Hammerschmidt KSA, Vargas MAO, Girondi JBR. Nursing staff knowledge in relation to complications of diabetes mellitus in emergency services. Acta Paul Enferm [Internet]. 2014 [acesso em: 30 jun.

2016];27(6):520-5. Disponível em: http://dx.doi.org/10.1590/1982-0194201400085.

10. Fontanella BJB, Luchesi BM, Saidel MGB, Ricas J, Turato ER, Melo DG. Amostragem em pesquisas qualitativas: proposta de procedimentos para constatar saturação teórica. Cad Saude Publica [Internet]. 2011 [acesso em: 30 jun. 2016];27(2):389-94. Disponível em: http://dx.doi.org/10.1590/S0102-311X2011000200020.

11. Minayo MCS. Análise qualitativa: teoria, passos e fidedignidade. Cien Saude Colet [Internet]. 2012 [acesso em: 30 jun. 2016];17(3):621-6. Disponível em: http://dx.doi.org/10.1590/S1413-81232012000300007.

12. Gerência de Ensino e Pesquisa, Grupo Hospitalar Conceição. Diretrizes Clínicas/Protocolos Assistenciais GEP-CHC. Manual Operacional [Internet]. Porto Alegre: Grupo Hospitalar Conceição; 2008 [acesso em: 30 jun. 2016]. Disponível em: https://www.nescon.medicina.ufmg.br/biblioteca/imagem/2247.pdf.

13. Dal Pai D, Lautert L. Work under urgency and emergency and its relation with the health of nursing professionals. Rev Lat Am Enfermagem [Internet]. 2008 [acesso em: 30 jun. 2016];16(3):439-44. Disponível em: http://dx.doi.org/10.1590/S0104-11692008000300017.

14. Garlet ER, Lima MADS, Santos JLG, Marques GQ. Organização do trabalho de uma equipe de saúde no atendimento ao usuário em situações de urgência e emergência. Texto Context - Enferm [Internet]. 2009 [acesso em: 30 jun. 2016];18(2):266-72. Disponível em: http://dx.doi.org/10.1590/S0104-07072009000200009.

15. Guidoni CM, Olivera CMX, Freitas O, Pereira LRL. Assistência ao diabetes no Sistema Único de Saúde: análise do modelo atual. Brazilian J Pharm Sci [Internet]. 2009 [acesso em: 30 jun. 2016];45(1):37-48. Disponível em: http://dx.doi.org/10.1590/S1984-82502009000100005.

16. Sociedade Brasileira de Diabetes. Controle da Hiperglicemia Intra-hospitalar em Pacientes Críticos e não Críticos. São Paulo: AC Farmacêutica, 2011.

17. Avelar AFM, Peterlini MAS, Onofre PSC, Pettengill MAM, Pedreira MLG. Capacitação de enfermeiros para uso da ultrassonografia na punção intravascular periférica. Acta Paul Enferm [Internet]. 2010 [acesso em: 30 jun.

2016];23(3):433-6. Disponível em: http://dx.doi.org/10.1590/S0103-21002010000300020. 
18. Faria HTG, Rodrigues FFL, Zanetti ML, Araújo MFM, Damasceno MMC. Factors associated with adherence to treatment of patients with diabetes mellitus. Acta Paul Enferm [Internet]. 2013 [acesso em: 30 jun. 2016];26(3):231-7. Disponível em: http://dx.doi.org/10.1590/S0103-21002013000300005.

19. Grossi SAA. O manejo da cetoacidose em pacientes com Diabetes Mellitus: subsídios para a prática clínica de enfermagem. Rev Esc Enferm USP [Internet]. 2006 [acesso em: 30 jun. 2016];40(4):582-6. Disponível em:

http://dx.doi.org/10.1590/S0080-62342006000400019.

20. Ministério da Saúde. acolhimento e classificação de risco nos serviços de urgência [Internet]. Brasília: Ministério da Saúde; 2009 [acesso em: 30 jun. 2016]. Disponível em:

http://bvsms.saude.gov.br/bvs/publicacoes/acolhimento_classificaao_risco servico_urgencia.pdf.

21. Souza AC, Bonilha ALL, Veronese AM. Buscando a integralidade no atendimento ao indivíduo diabético: do serviço de atendimento móvel de urgência à atenção primária à saúde. Ciência, Cuid e Saúde [Internet]. 2008 [acesso em: 30 jun. 2016];7(1):21-6. Disponível em: http://dx.doi.org/10.4025/cienccuidsaude.v7i1.4891.

22. Wilson V. Diagnosis and treatment of diabetic ketoacidosis. Emerg Nurse [Internet]. 2012 [acesso em: 30 jun. 2016];20(7):14-8. Disponível em: http://dx.doi.org/10.7748/en2012.11.20.7.14.c9405.

23. Santos AL, Cecílio HPM, Teston EF, Arruda GO, Peternella FMN, Marcon SS. Microvascular complications in type 2 diabetes and associated factors: a telephone survey of self-reported morbidity. Cien Saude Colet [Internet]. 2015 [acesso em: 30 jun. 2016];20(3):761-70. Disponível em: http://dx.doi.org/10.1590/1413-81232015203.12182014 24. Santos AL, Teston EF, Latorre MRDO, Mathias TAF, Marcon SS. Trend in hospitalizations for diabetes mellitus: implications for health care. Acta Paul Enferm [Internet]. 2015 [acesso em: 30 jun. 2016];28(5):401-7. Disponível em: http://dx.doi.org/10.1590/1982-0194201500068.

25. Cortez DN, Reis IA, Souza DAS, Macedo MML, Torres HC. Complications and the time of diagnosis of diabetes mellitus in primary care. Acta Paul Enferm [Internet]. 2015 [acesso em: 30 jun. 2016];28(3):250-5. Disponível em: http://dx.doi.org/10.1590/1982-0194201500042. 